Cahiers $d u$ MONDE RUSSE

\section{Cahiers du monde russe}

Russie - Empire russe - Union soviétique et États indépendants

42/2-4 | 2001

La police politique en Union soviétique, 1918-1953

\title{
Les documents du Ministère public de l'URSS comme sources de l'histoire du Goulag, 1945-1953.
}

Dina N. NOHOTOVIČ

\section{OpenEdition}

\section{Journals}

Édition électronique

URL : https://journals.openedition.org/monderusse/8467

DOI : 10.4000/monderusse.8467

ISSN : $1777-5388$

Éditeur

Éditions de l'EHESS

\section{Édition imprimée}

Date de publication : 1 avril 2001

Pagination : $549-556$

ISBN : 2-7132-1398-3

ISSN : $1252-6576$

Référence électronique

Dina N. NOHOTOVIČ, "Les documents du Ministère public de l'URSS comme sources de l'histoire du Goulag, 1945-1953. », Cahiers du monde russe [En ligne], 42/2-4 | 2001, mis en ligne le 01 janvier 2007, consulté le 04 septembre 2022. URL : http://journals.openedition.org/monderusse/8467 ; DOI : https://doi.org/10.4000/monderusse.8467 


\section{CAIR N}

chercher : repérer : avancer

Cet article est disponible en ligne à l'adresse :

http://www.cairn.info/article.php?ID REVUE=CMR\&ID NUMPUBLIE=CMR 422\&ID ARTICLE=CMR 4220549

Les documents du Ministère public de l'URSS comme sources de l'histoire
du Goulag, 1945-1953

par Dina N. NOHOTOVIC

| Editions de l'EHESS | Cahiers du monde russe

2001/2-3-4 - Vol 42

ISSN 1252-6576 | ISBN 2713213983 | pages 549 à 556

Pour citer cet article :

- N. NOHOTOVIC D., Les documents du Ministère public de l'URSS comme sources de l'histoire du Goulag, 1945-

1953, Cahiers du monde russe 2001/2-3-4, Vol 42, p. 549-556.

Distribution électronique Cairn pour les Editions de l'EHESS.

(C) Editions de l'EHESS. Tous droits réservés pour tous pays.

La reproduction ou représentation de cet article, notamment par photocopie, n'est autorisée que dans les limites des conditions générales d'utilisation du site ou, le cas échéant, des conditions générales de la licence souscrite par votre établissement. Toute autre reproduction ou représentation, en tout ou partie, sous quelque forme et de quelque manière que ce soit, est interdite sauf accord préalable et écrit de l'éditeur, en dehors des cas prévus par la législation en vigueur en France. Il est précisé que son stockage dans une base de données est également interdit. 


\section{LES DOCUMENTS DU MINISTÈRE PUBLIC DE L'URSS COMME SOURCES DE L'HISTOIRE DU GOULAG, 1945-1953}

Le Ministère public (Prokuratura) et le ministère des Affaires intérieures d'URSS (MVD) apparaissaient, dans le système punitif soviétique, comme deux structures concurrentes. En tant qu'organe de contrôle, le Ministère public a, dans de nombreux cas, empiété sur le champ d'activité du MVD. Ces ingérences avaient souvent pour but de faire apparaître des défaillances dans l'activité de certaines structures du MVD et de ses collaborateurs. Aussi des conflits liés aux intérêts opposés des deux ministères n'ont pas manqué de se produire lorsqu'ils ont dû coopérer. Pour les historiens, les documents qui reflètent ces conflits constituent une source d'étude très importante. Ce sont précisément ces matériaux qui leur permettent de remédier au caractère limité de l'information émanant d'une source restreinte (en l'occurrence, l'information du MVD) et de présenter plus objectivement la situation réelle.

La coopération entre le MVD et le Ministère public constitue un problème complexe, aux aspects multiples et requiert une étude spécifique. Cet article présente des observations liées à la surveillance exercée par le Ministère public sur le système des camps de détention dont la gestion dépendait du MVD. Il se fonde sur les matériaux provenant du Secrétariat du MVD, de la Direction centrale des camps (Glavnoe upravlenie lagerej - GULAG), du Département pénitentiaire du MVD, des départements de surveillance des lieux de détention et des affaires concernant les mineurs du Ministère public. Aujourd'hui, l'interdiction d'accès aux fonds du Ministère public a été levée, et concerne notamment les documents des deux derniers départements mentionnés ci-dessus. Les documents concernant le Goulag et le Département pénitentiaire sont aussi presque totalement accessibles, les documents du Secrétariat du MVD le sont partiellement. En fait, sont accessibles les normes qui réglementent l'organisation des répressions politiques et également ce que l'on appelle « le dossier spécial de Stalin », c'est-à-dire les copies des documents que le MVD remettait à Stalin. 
On peut classer en quatre groupes les documents du Ministère public concernant la situation dans les prisons et les camps, répartis dans de nombreux fonds : le premier comprend les rapports des procureurs des camps de rééducation par le travail concernant la façon dont étaient menées et contrôlées les enquêtes et les procédures judiciaires ; le deuxième les rapports concernant les travaux des Ministères publics des directions des camps de rééducation par le travail ; le troisième les notes rédigées par les procureurs des républiques et des régions (oblasti) sur les résultats des contrôles des conditions de détention et d'exploitation du travail des prisonniers ; le quatrième la correspondance entre le Ministère public et les organes du MVD concernant les camps et les prisons.

Chaque groupe avait, dans le système bureaucratique, sa propre sphère de diffusion. Il était de règle d'adresser les rapports émis par les procureurs des camps au responsable du Département de surveillance des lieux de détention du Ministère public d'URSS. L'information provenant des procureurs des républiques et des régions était envoyée au procureur général d'URSS ou à ses adjoints. Si les trois premiers groupes étaient surtout constitués de documents provenant des services internes des parquets, le quatrième témoignait de l'interaction entre le Ministère public et le MVD sur les questions posées par le Ministère public. La correspondance avec le MVD provenant du fonds du Ministère public d'URSS est comparativement peu volumineuse. Toutefois, un ensemble important de documents, portant sur la période 1945-1953, a été déposé dans le fonds documentaire du Secrétariat du MVD sous l'intitulé «Correspondance du MVD avec le Ministère public ». Dans l'ensemble, tous les documents transférés constituent une source précieuse pour la recherche sur différents aspects de la politique de répression de l'État stalinien.

Ces sources contiennent tout d'abord des informations importantes sur la situation dans les camps, les colonies et les prisons. À la différence des documents établis par les départements du MVD (le Goulag et le Département des prisons), qui tendaient à atténuer la gravité de nombreux problèmes, les matériaux fournis par les services du contrôle du Ministère public étaient généralement plus sincères et contenaient des informations sur des sujets qui n'étaient pas traités dans les comptes rendus des services du MVD. La direction du Ministère public d'URSS présentait régulièrement des plaintes de toutes sortes au sujet d'abus constatés au MVD. C'est ainsi que, le 25 décembre 1946, le procureur général d'URSS, K. P. Goršenin, a adressé au ministre de l'Intérieur, S. N. Kruglov, des informations détaillées qui prenaient en compte les résultats des contrôles des conditions de détention et d'exploitation du travail des détenus dans les colonies de rééducation par le travail. Les faits rapportés dressaient un tableau effrayant des conditions de vie des détenus. Au lieu de disposer d'une surface habitable de $2 \mathrm{~m}^{2}$ conformément aux instructions de la norme en vigueur, les détenus se contentaient d'environ 0,7$0,8 \mathrm{~m}^{2}$, ils n'avaient ni vêtements ni chaussures aussi ne les envoyait-on pas au travail ou alors les y envoyait-on nu-pieds, etc. Kruglov chargea ses adjoints Černišev et Nasedkin d'examiner ces faits ${ }^{1}$.

1. Gosudarstvennyj Arhiv Rossijskoj Federacii (GARF), collection de documents 
Cependant, les comptes rendus du procureur irritaient les responsables du MVD qui estimaient qu'il était beaucoup plus facile d'attirer l'attention sur les problèmes que de les résoudre. Dans le cadre de la mission dont l'avait chargé Kruglov, Černišev prépara le projet de réponse suivant à Goršenin :

\begin{abstract}
«Afin de réduire des correspondances superflues, et qui ne sont utiles à personne, entre le MVD et le Ministère public, nous estimons indispensable que les procureurs locaux participent aux réunions opérationnelles auprès du ministre et du responsable de la Direction du MVD où l'on débat des bilans des tournées d'inspections mensuelles dans les prisons et les colonies [...]. Ainsi, les procureurs locaux, à l'aide de leurs documents, pourraient faire en sorte que ces réunions stratégiques soient plus efficaces que de longs échanges de correspondance au sujet des défauts constatés dans le semestre écoulé. $\gg^{2}$
\end{abstract}

Bien que la réponse de Kruglov envoyée en fin de compte ne fût pas aussi catégorique, on pouvait cependant y percevoir son mécontentement et l'accent y était porté sur les performances du MVD.

Cette réaction des dirigeants du MVD à l'ingérence des procureurs, à en juger d'après les documents disponibles, était tout à fait typique. Quelques mois plus tard à peine, en février 1947, le nouveau procureur général d'URSS, G. N. Safonov, informa le MVD des violations du règlement concernant l'envoi de détenus au camp de Pétchora du Nord (Severo-Pečora), et plus spécialement de l'envoi dans ce camp de détenus en mauvaise santé, qui ne pouvaient être employés dans les camps où les conditions de travail étaient particulièrement pénibles. La réponse de Černišev fut cette fois encore très vive : « La question du travail de ceux qui se trouvent dans les camps de rééducation est certainement un élément très important dans l'activité économique des camps, mais en même temps elle n'apparaît pas comme essentielle dans le contrôle du Ministère public sur les lieux de détention. $»^{3}$

De leur côté les dirigeants du MVD attaquaient périodiquement le Ministère public, exploitant les aspects les plus vulnérables de son activité, c'est-à-dire la violation des délais d'enquêtes par les procureurs. Ainsi, en mai 1946, le viceministre de l'Intérieur, V. S. Rjasnoj, envoya une lettre au Ministère public dans laquelle il réclamait l'accélération de l'examen des affaires qui devaient être étudiées lors d'une Assemblée extraordinaire du MVD, motivant sa demande par le fait que le Ministère Public avait par-devers lui 1020 dossiers, envoyés par les organes périphériques à l'Assemblée extraordinaire. En août de la même année, Kruglov, dans une note à Goršenin, attira l'attention sur le fait que, sur les 8400 détenus dans les prisons relevant du Ministère public, 2951 étaient détenus en violation des délais accordés par la loi pour la conduite de l'enquête ${ }^{4}$. On n'a pas
2. Ibid
3. Ibid.
4. Ibid. 
trouvé de documents qui auraient permis de connaître la réaction des procureurs à ces interventions. Cependant, tous ces exemples témoignent bien des relations conflictuelles qui existaient entre les deux administrations dans la première période de l'après-guerre.

Par ailleurs, certains faits démontrent que le MVD et le Ministère public ont progressivement instauré un style de relations basé sur le compromis, préférant éviter des conflits qui, en fin de compte, pouvaient être préjudiciables aux deux ministères. On peut considérer comme un signe de cet « armistice » bureaucratique le fait que le procureur général n'adressait pas systématiquement au MVD les rapports, préparés par le Ministère public, concernant l'activité du MVD. La comparaison des archives du Ministère public et du MVD en témoigne. Dans plusieurs cas, la direction du Ministère public n'a donné aucune suite aux propositions de ses fonctionnaires lorsqu'elles traitaient de problèmes très généraux ne relevant pas vraiment de l'action des services du MVD mais plus largement de la politique gouvernementale. On peut citer à titre d'exemple le rapport du 3 octobre 1947 du responsable de la Direction des lieux de détention du Ministère public d'URSS consacré aux conditions de vie et au régime de détention des condamnés aux travaux forcés 5 . Ce rapport soulevait un problème très important : seuls $7 \%$ des bagnards étaient reconnus aptes aux travaux forcés les plus pénibles physiquement, $39 \%$ pouvaient effectuer des travaux légers et plus de $26 \%$ étaient considérés comme inaptes. Ces chiffres remettaient évidemment en question la raison d'être de l'institution des travaux forcés elle-même, ce qui sortait du cadre des compétences du MVD et du Ministère public. Le rapport, à en juger d'après les documents que nous avons étudiés, ne fut pas pris en considération.

Comme en témoignent les documents du Secrétariat du MVD, le Ministère public se montra particulièrement actif dans le domaine du contrôle des camps entre 1948 et 1950. Cela ne signifie pas que l'opposition entre ces deux administrations se soit renforcée. Dans de nombreux cas, il s'agissait d'exécuter des mesures dont la responsabilité incombait à la fois au MVD et au Ministère public, ce qui présupposait qu'ils agissent de façon conjointe et concertée. Ce fut le cas par exemple lors de la promulgation du décret du Présidium du Soviet Suprême d'URSS le 22 avril 1949 : «De la levée des peines des femmes enceintes et des femmes ayant de jeunes enfants». Le MVD et le Ministère public qui étaient chargés de l'application pratique du décret travaillèrent en bonne intelligence à la prorogation des délais de sa mise en œuvre ${ }^{6}$. Le fait qu'ils soient responsables conjointement de la réduction de la criminalité à l'intérieur des camps a prédéterminé le caractère non conflictuel des contrôles exercés par le Ministère public lors de l'éclatement de troubles généralisés dans les camps, d'actes de banditisme, de meurtres de détenus, etc.

5. GARF, f. R-8131, op. 37, d. 3817, 11. 9-26.

6. Ibid. 
Dans de nombreux cas, les positions du MVD et du Ministère public quant aux questions-clés de la politique pénitentiaire coïncidaient pleinement. Il en fut ainsi par exemple lorsqu'ils réglementèrent le « décompte des jours de travail ». Suivant ce système, le temps de détention des condamnés, quelle que fût leur catégorie, pouvait être substantiellement abrégé (par exemple, si on faisait du bon travail, trois journées de détention dans le camp comptaient pour quatre, diminuant ainsi le temps de détention d'un quart). Bien que le décompte des jours de travail eût été supprimé en 1939 à la demande de Berija, alors commissaire du peuple aux Affaires intérieures, on commença, à la fin de la guerre, à remettre ces décomptes en vigueur dans des sites prioritaires pour le MVD. Le système des décomptes constituait en effet le facteur le plus efficace et le plus réaliste pour accroître le niveau de productivité du travail des condamnés. Tout en l'admettant, la direction du MVD voyait sans enthousiasme le rétablissement du système des décomptes, elle craignait une libération anticipée massive de détenus et un abaissement du niveau du volume de la main-d'œuvre dans l'économie relevant des travaux forcés. Le MVD reçut toutefois sur cette question le soutien total du Ministère public. En juin 1950, Safonov envoya à Kruglov un courrier spécial selon lequel le Ministère public jugeait nécessaire d'arrêter toute nouvelle extension de la liste des camps où s'appliquait le décompte des jours de travail. Le 7 juillet 1950, lors d'une réunion de la direction du MVD où ce courrier fut examiné, il fut déclaré que le MVD « estimait lui aussi qu'il n'était pas rationnel de soutenir les requêtes des organisations revendiquant l'extension du nombre de camps appliquant les décomptes des jours de travail des détenus ». Très vite, un courrier dans ce sens, signé de Serov, fut adressé à Safonov ${ }^{7}$.

Des tendances marquées « de compréhension mutuelle » ont prévalu également lors de l'examen du fonctionnement des camps spéciaux créés pour la détention des « détenus particulièrement dangereux » sur la base d'une résolution du Conseil des ministres du 21 février 1948. Le contrôle de ces camps constituait l'un des principaux axes de l'activité du Ministère public dans les années 1950-1952. Les documents du Ministère apparaissent à cet égard comme une source importante pour l'étude des camps spéciaux considérés comme une subdivision du système des camps.

Ainsi, le 23 mai 1950, le procureur général Safonov adressa au ministre de l'Intérieur, Kruglov, une note circonstanciée établie à partir des documents de contrôle de 56 procureurs en charge des camps de rééducation par le travail. Cette information, de façon générale, faisait état de violations des règles d'isolation des détenus, condamnés « comme criminels particulièrement dangereux ». Il en ressortait que, peu de temps après la création des camps spéciaux, 3636 détenus y furent inculpés de banditisme, brigandage ou meurtres avec préméditation accomplis à l'intérieur même des camps ${ }^{8}$. Étant donné que la direction du MVD elle-même reconnaissait l'état critique dans lequel se trouvaient les camps spéciaux et la 
lenteur de leur mise en place, la réponse du vice-ministre de l'Intérieur, I. A. Serov, se présenta sous la forme d'une note analytique détaillée qui reprenait point par point les remarques du Ministère public ${ }^{9}$.

Les contrôles effectués par les procureurs en 1951-1952 dans les camps de Noril'sk, de Vorkuta, de Pétchora du Nord, d'Ivdel', de Bodajbo, de Sibir', de Kuneevo firent l'objet d'examens tout aussi impartiaux. Les bilans des contrôles effectués dans le camp de Bodajbo, dont les détenus exploitaient les mines d'or, furent même transmis à Stalin. En 1952, plusieurs contrôles effectués au camp de Vorkuta furent soumis à la direction du MVD pour y être examinés par ses membres $^{10}$.

Si l'on se fonde sur les documents appartenant aux fonds du MVD et du Ministère public d'URSS, on peut dire que les rapports qui se sont instaurés entre ces deux administrations lors de l'examen des questions liées au développement du système pénitentiaire à la fin des années 40 relèvent davantage de la coopération que de la rivalité. Le MVD s'est accommodé des prétentions du Ministère public dans le contrôle des camps. Toutefois les procureurs eux-mêmes, lorsqu'ils rendaient leur jugement, mesuraient leurs critiques, limitant leurs remarques et leurs propositions au strict minimum. En dépit de la conduite correcte des procureurs, la direction du MVD appréciait peu leurs efforts pour élargir le système déjà suffisamment complexe de contrôle des camps par le Ministère public. Le jugement porté en 1950-1951 sur le projet d'instruction : «Du contrôle par le Ministère public du respect de la légalité dans les lieux de détention », élaboré par ce même ministère, en témoigne. La grande innovation de ce projet était la disposition selon laquelle on conférait aux procureurs des villes et des arrondissements (rajony) le droit de contrôler les lieux de détention du MVD. Dans les instances du MVD où le projet d'instruction fut envoyé pour accord, les réponses furent catégoriquement négatives, sous prétexte que l'extension des droits des procureurs des villes et des arrondissements affaiblirait le régime du secret, entraînerait la diffusion des données concernant le nombre et la composition des détenus, ainsi que d'autres renseignements relevant du secret d'État ${ }^{11}$. Le bannissement au secret était un procédé auquel la direction du MVD recourait dans les cas où elle cherchait à limiter le droit d'accès des contrôleurs dans les camps. La véritable cause du refus n'était pas, dans ce cadre, de préserver le secret puisque, en fin de compte, celui-ci reposait sur les procureurs, mais de tenter d'empêcher l'élargissement des fonctions de contrôle du Ministère public.

Cette étude montre que, en marge des documents du NKVD-MVD largement exploités par les historiens depuis quelques années, les matériaux provenant du
9. Ibid.
10. Ibid.
11. Ibid. 
Ministère public d'URSS constituent une source importante pour l'étude de la politique répressive et du système carcéral et pénitentiaire. Bien que le Ministère public n'ait pas exercé une influence déterminante sur les processus fondamentaux dans ce domaine, les matériaux résultant des contrôles des procureurs et les expertises préalables aux actes normatifs contiennent des données précieuses qui témoignent de la situation des détenus et des mécanismes de la politique de répression.

(Traduit du russe par Françoise Cordes-Baudrillard et Jacqueline Tordjman)

Gosudarstvennyj Arhiv Rossijskoj Federacii

ul. Bol`̌saja Pirogovskaja, 17

$119817 \mathrm{Moscou}$ 